PROCEEDINGS OF THE

AMERICAN MATHEMATICAL SOCIETY

Volume 138, Number 2, February 2010, Pages 455-459

S 0002-9939(09)10101-6

Article electronically published on September 16, 2009

\title{
A SHORT PROOF OF HRS-TILTING
}

\author{
XIAO-WU CHEN
}

(Communicated by Birge Huisgen-Zimmermann)

\begin{abstract}
We give a short proof of the tilting theorem by Happel, Reiten and Smalø: two abelian categories $\mathcal{A}$ and $\mathcal{B}$ are derived equivalent, provided that $\mathcal{B}$ is tilted from $\mathcal{A}$.
\end{abstract}

\section{INTRODUCTION}

Let $\mathcal{A}$ be an abelian category. Recall that a torsion pair on $\mathcal{A}$ is a pair $(\mathcal{T}, \mathcal{F})$ of full additive subcategories satisfying the following conditions:

(T1) $\operatorname{Hom}_{\mathcal{A}}(T, F)=0$ for all $T \in \mathcal{T}$ and $F \in \mathcal{F}$;

(T2) for each object $A \in \mathcal{A}$, there exists a short exact sequence

$$
0 \longrightarrow T \longrightarrow A \longrightarrow F \longrightarrow 0
$$

such that $T \in \mathcal{T}$ and $F \in \mathcal{F}$.

Given a torsion pair $(\mathcal{T}, \mathcal{F})$, it follows that the subcategory $\mathcal{T}$ is closed under extensions and factor objects; the subcategory $\mathcal{F}$ is closed under extensions and subobjects. In particular, both subcategories $\mathcal{T}$ and $\mathcal{F}$ are closed under direct summands. A torsion pair $(\mathcal{T}, \mathcal{F})$ is called a tilting torsion pair, provided that each object in $\mathcal{A}$ embeds into an object in $\mathcal{T}$. Dually a torsion pair $(\mathcal{T}, \mathcal{F})$ is called a cotilting torsion pair, provided that each object in $\mathcal{A}$ is a factor object of an object in $\mathcal{F}([4$, Chapter I, section 3]).

Let $X=\left(X^{n}, d_{X}^{n}\right)_{n \in \mathbb{Z}}$ be a complex in $\mathcal{A}$, where $d_{X}^{n}: X^{n} \rightarrow X^{n+1}$ are the differential morphisms satisfying $d_{X}^{n+1} \circ d_{X}^{n}=0$; its shift $X[1]$ is a complex given by $(X[1])^{n}=X^{n+1}$ and $d_{X[1]}^{n}=-d_{X}^{n+1}$. Denote by $H^{n}(X)$ the $n$-th cohomology of $X$. Denote by $\mathbf{D}(\mathcal{A})$ the (unbounded) derived category of $\mathcal{A}$, and denote by $\mathbf{D}^{+}(\mathcal{A}), \mathbf{D}^{-}(\mathcal{A})$ and $\mathbf{D}^{b}(\mathcal{A})$ the full subcategories of $\mathbf{D}(\mathcal{A})$ consisting of boundedbelow, bounded-above and bounded complexes, respectively $(8,5])$. We will always identify the abelian category $\mathcal{A}$ as the full subcategory of $\mathbf{D}(\mathcal{A})$ consisting of stalk complexes concentrated in degree zero $([5$, p. 40, Proposition 4.3]). Let $(\mathcal{T}, \mathcal{F})$ be a torsion pair on $\mathcal{A}$. Following [4, Chapter I, section 2], denote by $\mathcal{B}$ the full subcategory of $\mathbf{D}(\mathcal{A})$ consisting of complexes $X$ such that $H^{0}(X) \in \mathcal{T}, H^{-1}(X) \in \mathcal{F}$

Received by the editors February 11, 2009, and, in revised form, April 27, 2009, and May 29, 2009 .

2000 Mathematics Subject Classification. Primary 18G35; Secondary 18E30.

This project was supported by Alexander von Humboldt Stiftung and was also partially supported by the China Postdoctoral Science Foundation (Nos. 20070420125 and 200801230). The author also gratefully acknowledges the support of the K.C. Wong Education Foundation, Hong Kong.

(C)2009 American Mathematical Society Reverts to public domain 28 years from publication 
and $H^{n}(X)=0$ for $n \neq 0,-1$. Then it follows that $\mathcal{T} \subseteq \mathcal{B}$ and $\mathcal{F}[1] \subseteq \mathcal{B}$. By [4, Chapter I, Proposition 2.1] the category $\mathcal{B}$ is the heart of a $t$-structure on $\mathbf{D}(\mathcal{A})$, and thus by [1, Théorème 1.3.6] it is an abelian category (also see [3. Chapter IV, $\S 4$, Theorem 4]); moreover, the pair $(\mathcal{F}[1], \mathcal{T})$ is a torsion pair on $\mathcal{B}$ (4, Chapter I, Corollary 2.2]).

One might expect that the resulting new abelian category $\mathcal{B}$ is derived equivalent to $\mathcal{A}$. However, by the example in [4, p. 16] this is in general false. Happel, Reiten and Smalø have shown the following remarkable result (4, Chapter I, Theorem 3.3]).

Theorem (Happel-Reiten-Smal $\varnothing)$. Let $(\mathcal{T}, \mathcal{F})$ be a tilting torsion pair on $\mathcal{A}$. Then we have a triangle equivalence $\mathbf{D}(\mathcal{B}) \simeq \mathbf{D}(\mathcal{A})$ which is compatible with the inclusion of $\mathcal{B}$ into $\mathbf{D}(\mathcal{A})$. Similar results hold for $\mathbf{D}^{*}(-)$ with $* \in\{+,-, b\}$.

In the case of the theorem, the abelian category $\mathcal{B}$ is said to be tilted from $\mathcal{A}$. Note that the original statement of the theorem is about the equivalence between the bounded derived categories and requires the existence of enough projective or injective objects. The version given here was improved by Bondal and Van den Bergh [2, Proposition 5.4.3] and independently by Noohi (7, Theorem 7.6]). We will give a short proof of the theorem via an explicit construction of the equivalences.

\section{Proof of Theorem}

Throughout, $(\mathcal{T}, \mathcal{F})$ is a torsion pair on $\mathcal{A}$, and $\mathcal{B}$ is the resulting abelian category as above.

We have the following observation.

Lemma 2.1. Let $T=\left(T^{n}, d_{T}^{n}\right)_{n \in \mathbb{Z}}$ be a complex such that each term belongs to $\mathcal{T}$. Then it is acyclic in $\mathcal{A}$ if and only if it is acyclic in $\mathcal{B}$.

Proof. To prove the "only if" part of the lemma, we assume that the complex $T$ is acyclic in $\mathcal{A}$. Since $\mathcal{T}$ is closed under factor objects in $\mathcal{A}$, the complex $T$ splits into short exact sequences in $\mathcal{A}$,

$$
\xi^{n}: 0 \longrightarrow T^{\prime n} \stackrel{i^{n}}{\longrightarrow} T^{n} \stackrel{p^{n}}{\longrightarrow} T^{\prime n+1} \longrightarrow 0
$$

such that $T^{\prime n} \in \mathcal{T}$ and $d_{T}^{n}=i^{n+1} \circ p^{n}$. Since $\mathcal{B}$ is the heart of a $t$-structure on $\mathbf{D}(\mathcal{A})$, a sequence $0 \rightarrow B^{0} \stackrel{f}{\rightarrow} B^{1} \stackrel{g}{\rightarrow} B^{2} \rightarrow 0$ in $\mathcal{B}$ is short exact if and only if there is a triangle $B^{0} \stackrel{f}{\rightarrow} B^{1} \stackrel{g}{\rightarrow} B^{2} \rightarrow B^{0}[1]$ in $\mathbf{D}(\mathcal{A})$ (11, 1.3] and [3, Chapter IV, $\S 4])$. Note that short exact sequences in $\mathcal{A}$ induce triangles in $\mathbf{D}(\mathcal{A})$ ([5, p. 62, Proposition 6.1]). Hence each $\xi^{n}$ becomes a short exact sequence in $\mathcal{B}$. Thus by splicing $\xi^{n}$ together we obtain that $T$ is acyclic in $\mathcal{B}$. Recall that the pair $(\mathcal{F}[1], \mathcal{T})$ is a torsion pair on $\mathcal{B}$, and thus one proves the "if" part of this lemma similarly.

We will need the following result.

Lemma 2.2 (4, Chapter I, Proposition $3.2(\mathrm{i})])$. Let $(\mathcal{T}, \mathcal{F})$ be a torsion pair on $\mathcal{A}$ and let $\mathcal{B}$ be as above. If the torsion pair $(\mathcal{T}, \mathcal{F})$ is tilting, then the torsion pair $(\mathcal{F}[1], \mathcal{T})$ on $\mathcal{B}$ is cotilting.

Remark 2.3. Note that the converse of the above lemma holds true as stated in 4. Chapter I, Proposition 3.2 (i)]. However, it seems to the author that a dual argument of this lemma does not work. Instead, thanks to the Happel-ReitenSmalø Theorem and then by combining [4, Chapter I, Proposition 3.4] with the 
"only if" part of [4, Chapter I, Proposition 3.2 (ii)], one obtains the converse of Lemma 2.2. (To see [4, Chapter I, Proposition 3.4] in our situation, one uses the fact that the equivalence in the above Theorem is compatible with the inclusion of $\mathcal{B}$ into $\mathbf{D}(\mathcal{A})$.)

Proof of Theorem. Denote by $\mathbf{K}(\mathcal{A})$ the homotopy category of complexes in $\mathcal{A}$, and denote by $\mathbf{K}(\mathcal{T})$ (resp. $\mathbf{K}_{\mathrm{ac}}(\mathcal{A})$ ) its full subcategory consisting of complexes in $\mathcal{T}$ (resp. acyclic complexes). The inclusion $\mathbf{K}(\mathcal{T}) \hookrightarrow \mathbf{K}(\mathcal{A})$ induces the triangle functor

$$
F: \mathbf{K}(\mathcal{T}) / \mathbf{K}(\mathcal{T}) \cap \mathbf{K}_{\mathrm{ac}}(\mathcal{A}) \longrightarrow \mathbf{D}(\mathcal{A}) .
$$

Since the torsion pair $(\mathcal{T}, \mathcal{F})$ is tilting and $\mathcal{T}$ is closed under factor objects, we have for each object $A \in \mathcal{A}$ a short exact sequence $0 \rightarrow A \rightarrow T^{0} \rightarrow T^{1} \rightarrow 0$ such that each $T^{i} \in \mathcal{T}$. Note further that the subcategory $\mathcal{T}$ is closed under extensions, so we infer that the conditions in [5, p. 42, Lemma 4.6, 2)] are fulfilled, and thus for each complex $X$ in $\mathbf{K}(\mathcal{A})$ there is a quasi-isomorphism $X \rightarrow T$ such that $T \in \mathbf{K}(\mathcal{T})$. This implies that the functor $F$ is dense, and by [8, p. 283, 4-2 Théorème] it is also fully faithful; that is, the functor $F$ is an equivalence and thus a triangle equivalence ([6. Lemma 8.2]). By Lemma 2.2 we may apply a similar argument to obtain the triangle equivalence

$$
G: \mathbf{K}(\mathcal{T}) / \mathbf{K}(\mathcal{T}) \cap \mathbf{K}_{\mathrm{ac}}(\mathcal{B}) \longrightarrow \mathbf{D}(\mathcal{B})
$$

By Lemma 2.1 we have $\mathbf{K}(\mathcal{T}) \cap \mathbf{K}_{\mathrm{ac}}(\mathcal{A})=\mathbf{K}(\mathcal{T}) \cap \mathbf{K}_{\mathrm{ac}}(\mathcal{B})$. Hence the composite functor $F G^{-1}: \mathbf{D}(\mathcal{B}) \rightarrow \mathbf{D}(\mathcal{A})$ is the desired triangle equivalence, where $G^{-1}$ denotes a quasi-inverse of $G$.

To see the other equivalences, let $* \in\{+,-, b\}$ and let $\mathbf{K}^{*}(-)$ denote the corresponding homotopy category. Note that in the argument above, for a complex $X \in \mathbf{K}^{*}(\mathcal{A})$ we may find a quasi-isomorphism $X \rightarrow T$ such that $T \in \mathbf{K}^{*}(\mathcal{T})$. (For the case $*=+$, just consult the proof in [5, p. 43, 1)]; for the case $*=-$, because $\mathcal{T}$ is closed under factor objects, one may replace $T$ by its good truncations; for the case $*=b$, consult the proof in [5, p. 43, 1)] and note that since $\mathcal{T}$ is closed under factor objects, the argument therein is done within finitely many steps, and consequently the obtained complex $T$ is bounded.) Thus we can construct the triangle equivalences $F^{*}$ and $G^{*}$ as above, and then the composite functor $F^{*}\left(G^{*}\right)^{-1}: \mathbf{D}^{*}(\mathcal{B}) \rightarrow \mathbf{D}^{*}(\mathcal{A})$ is the desired triangle equivalence.

It remains to show that the triangle equivalence $F G^{-1}$ is compatible with the inclusion $\mathcal{B} \hookrightarrow \mathbf{D}(\mathcal{A})$. This is a little bit subtle. Let $B \in \mathcal{B}$ be an object. By Lemma 2.2 the torsion pair $(\mathcal{F}[1], \mathcal{T})$ is cotilting, and thus we have a short exact sequence in $\mathcal{B}$,

$$
\eta: 0 \longrightarrow T^{-1} \stackrel{d}{\longrightarrow} T^{0} \stackrel{g}{\longrightarrow} B \longrightarrow 0,
$$

such that each $T^{i} \in \mathcal{T}$. Hence by [1, 1.3] we obtain a triangle $\xi: T^{-1} \stackrel{d}{\rightarrow} T^{0} \stackrel{g}{\rightarrow}$ $B \rightarrow T^{-1}[1]$ in $\mathbf{D}(\mathcal{A})$. Note that by the explicit construction above, $F G^{-1}(B)$ is isomorphic to the complex $T=\cdots \rightarrow 0 \rightarrow T^{-1} \stackrel{d}{\rightarrow} T^{0} \rightarrow 0 \rightarrow \cdots$. Note further that the complex $T$ is the mapping cone of $d: T^{-1} \rightarrow T^{0}$, and thus we have a natural triangle $\beta$ : $T^{-1} \stackrel{d}{\rightarrow} T^{0} \rightarrow T \rightarrow T^{-1}$ [1] in $\mathbf{D}(\mathcal{A})$ ([6, Example 7.1]). By comparing the triangles $\xi$ and $\beta$ we infer that $T$ is isomorphic to $B$ ([5, p. 23, Proposition 1.1, c)]); in particular, the complex $T$ belongs to $\mathcal{B}$. By applying [1, 1.3] again, we obtain from the triangle $\beta$ the following short exact sequence in $\mathcal{B}$ :

$$
\gamma: 0 \longrightarrow T^{-1} \stackrel{d}{\longrightarrow} T^{0} \longrightarrow T \longrightarrow 0 .
$$


By comparing the short exact sequences $\eta$ and $\gamma$, we deduce a unique isomorphism $\theta_{B}: B \simeq T$ in $\mathcal{B}$. We claim that $\theta$ is natural in $B$. Then we have a natural isomorphism between the inclusion functor $\mathcal{B} \hookrightarrow \mathbf{D}(\mathcal{A})$ and the composite functor $\mathcal{B} \hookrightarrow \mathbf{D}(\mathcal{B}) \stackrel{F G^{-1}}{\longrightarrow} \mathbf{D}(\mathcal{A})$ (here we identify the complex $T$ with $F G^{-1}(B)$ ), and thus we are done.

To see the claim, let $f: B \rightarrow B^{\prime}$ be a morphism in $\mathcal{B}$. Choose a short exact sequence $\eta^{\prime}: \quad 0 \rightarrow T^{\prime-1} \stackrel{d^{\prime}}{\rightarrow} T^{\prime 0} \stackrel{g^{\prime}}{\rightarrow} B^{\prime} \rightarrow 0$ such that each $T^{i} \in \mathcal{T}$. Form the complex $T^{\prime}$, and then obtain the short exact sequence $\gamma^{\prime}$ and the isomorphism $\theta_{B^{\prime}}$ as above. Identify $G(T)$ with $B$, and $G\left(T^{\prime}\right)$ with $B^{\prime}$. Since the functor $G$ is fully faithful, we have a chain map $\phi: T \rightarrow T^{\prime}$ such that $G(\phi)=f$. This implies the following commutative exact diagram in $\mathcal{B}$ :

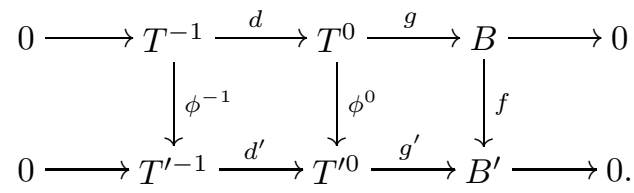

Then it follows immediately that $\theta_{B^{\prime}} \circ f=\phi \circ \theta_{B}$ in $\mathcal{B}$, and thus in $\mathbf{D}(\mathcal{A})$. This completes the proof.

Remark 2.4. The author is indebted to Bernhard Keller, who pointed out the following observation: one might have a better understanding of the proof above by using the notion of derived category of an exact category. In fact, note that the subcategory $\mathcal{T}$ is closed under extensions both in $\mathcal{A}$ and $\mathcal{B}$, and by Lemma 2.1, $\mathcal{T}$ inherits the same exact structure from that of $\mathcal{A}$ and $\mathcal{B}$. Denote by $\mathbf{D}(\mathcal{T})$ the derived category of the exact category $\mathcal{T}$ (for details, see [6, section 11]). Then by using a slight modification of [6. Theorem 12.1] or by the proof above, one obtains triangle equivalences $\mathbf{D}(\mathcal{T}) \simeq \mathbf{D}(\mathcal{A})$ and $\mathbf{D}(\mathcal{T}) \simeq \mathbf{D}(\mathcal{B})$, and thus one deduces the equivalence in the Happel-Reiten-Smalø Theorem (cf. [2, 5.4]).

\section{ACKNOWLEDGEMENTS}

The author would like to thank the referee for helpful comments. The author is grateful to Bernhard Keller, Henning Krause and Michel Van den Bergh for their help.

\section{REFERENCES}

1. A. Beilinson, J. Bernstein, P. Deligne, Faisceaux pervers, Astérisque 100, Société Mathématique de France, Paris, 1982. MR751966 (86g:32015)

2. A. Bondal, M. Van den Bergh, Generators and representability of functors in commutative and noncommutative geometry, Moscow Mathematical Journal 3 (2003), 1-36. MR1996800 (2004h:18009)

3. S.I. Gelfand, Yu I. Manin, Methods of Homological Algebra, Springer-Verlag, Berlin, 1996. MR.1438306 (97j:18001)

4. D. Happel, I. Reiten, S.O. Smalø, Tilting in Abelian Categories and Quasitilted Algebras, Memoirs Amer. Math. Soc., vol. 120, no. 575, Providence, Rhode Island, 1996. MR.1327209 (97j:16009)

5. R. Hartshorne, Residues and Duality, Lecture Notes in Math., 20, Springer-Verlag, BerlinNew York, 1966. MR 0222093 (36:5145)

6. B. Keller, Derived categories and their uses, Handbook of Algebra, 1, North-Holland, Amsterdam, 1996, 671-701. MR 1421815 (98h:18013) 
7. B. Noohi, Explicit HRS-tilting, Journal of Noncommutative Geometry 3(2) (2009), 223-259. MR2486794

8. J.L. Verdier, Catégories dérivées, état 0, Lecture Notes in Math., 569, Springer-Verlag, BerlinNew York, 1977, 262-311. MR0463174 (57:3132)

Department of Mathematics, University of Science and Technology of China, Hefei 230026, People's Republic of China

Current address: Institut für Mathematik, Universität Paderborn, 33095, Paderborn, Germany

E-mail address: xwchen@mail.ustc.edu.cn 